\title{
Perfil e evolução da mortalidade por causas externas em Joinville (SC), 2003 a 2016
}

\section{Profile and trend in mortality due to external causes in Joinville (SC) from 2003 to 2016}

\author{
Silvana Cardoso' (D), Maria Helena da Costa Naumann Gaertner ${ }^{1}$ (D), Luciane Haritsch ${ }^{1}$ (D), \\ Elisa Henning 2 (D), Maria Volpato Kropiwiec ${ }^{3}$ (D), Selma Cristina Franco' (D) \\ 'Universidade da Região de Joinville (Univille) - Joinville (SC), Brasil. \\ ${ }^{2}$ Departamento de Matemática, Universidade do Estado de Santa Catarina (UDESC) - Joinville (SC), Brasil. \\ ${ }^{3}$ Secretaria Municipal de Saúde de Joinville, Gerência de Vigilância em Saúde - Joinville (SC), Brasil.
}

Como citar: Cardoso S, Gaertner MHCN, Haritsch L, Henning E, Kropiwiec MV, Franco SC. Perfil e evolução da mortalidade por causas externas em Joinville (SC), 2003 a 2016. Cad Saúde Colet, 2020;28(2):189-200. https://doi.org/10.1590/1414462X202028020115

\begin{abstract}
Resumo
Introdução: A mortalidade por causas externas está entre as principais causas de óbito no mundo, com acentuada expressão em faixas etárias jovens. Objetivo: Descrever o perfil das mortes por causas externas em município do Sul do país e verificar sua tendência nos últimos anos. Método: Estudo retrospectivo dos óbitos por causas externas usando os coeficientes de mortalidade por sexo, grupo etário e causa da morte com análise por regressão quasi Poisson. Resultados: O coeficiente de mortalidade foi de 46,3 por 100 mil habitantes, predominando os acidentes por transporte terrestre (40\%) e agressões (30,5\%). Identificou-se aumento de $53 \%$ nos coeficientes de mortalidade, com variações segundo sexo, grupo etário e tipo. Conclusão: Necessitam-se intensificar políticas públicas conforme as especificidades na determinação dos tipos de morte em cada grupo etário.
\end{abstract}

Palavras-chave: mortalidade; causas externas; violência; prevenção de acidentes.

\begin{abstract}
Background: Mortality due to external causes is among of the leading causes of death worldwide, with great expression in young age groups. Objective: To describe the profile of deaths from external causes in a southern municipality of Brazil and verify its trend in the last years. Method: It was carried out a retrospective study of external causes deaths using mortality rates by gender, age group and cause of with quasi Poisson regression analysis. Results: Mortality coefficient was 46 per 100,000 inhabitants prevailing transport accidents (40\%) and aggressions (30.5\%). Increase of $53 \%$ in mortality rate was detected with variations by gender, age group and type. Conclusion: Social policies are necessary encompassing intersectoral actions and taking into account the specificities in determination of each age group's types of death.
\end{abstract}

Keywords: mortality; external causes; violence; accident prevention.

Trabalho realizado na Universidade da Região de Joinville (UNIVILLE) - Joinville (SC), Brasil.

Correspondência: Selma Cristina Franco. E-mail: scfranco@terra.com.br

Fonte de financiamento: nenhuma.

Conflito de interesses: nada a declarar.

Recebido em: Mar. 15, 2018. Aprovado em: Ago. 04, 2019

Este é um artigo publicado em acesso aberto (Open Access) sob a licença Creative Commons distribuição e reprodução em qualquer meio, sem restrições desde que o trabalho original seja corretamente citado. 


\section{INTRODUÇÃO}

A mortalidade por causas externas constitui um grave problema de saúde pública e está entre as principais causas de óbito no mundo, com acentuada expressão em faixas etárias jovens ${ }^{1}$. Em 2004, correspondeu a 10\% da mortalidade mundial e foi a terceira maior causa de óbito na população brasileira entre 2002 e 2015, atrás apenas de doenças do aparelho circulatório e de neoplasias ${ }^{2,3}$.

De 1990 a 2011, a taxa de mortalidade por causas externas no estado de Santa Catarina foi de 65,8 por 100 mil, situando-se abaixo da taxa nacional e das regiões Sul, Sudeste e Centro-Oeste e acima das regiões Norte e Nordeste.

A mortalidade por causas externas compreende diversos tipos de ocorrências e encontra-se categorizada no capítulo XX da Décima Revisão da Classificação Estatística Internacional de Doenças e Problemas Relacionados à Saúde (CID-10). Esse indicador não apenas reflete aspectos culturais e de desenvolvimento socioeconômico com diversos fatores de risco específicos, para cada tipo de acidente ou violência, como também expressa as condições da assistência médica ofertada e a qualidade do registro das ocorrências ${ }^{4}$. Ao refletir distintas realidades regionais, o estudo dessa mortalidade desagregada em áreas menores como os municípios ou regiões de saúde, torna-se relevante para possibilitar o estabelecimento de políticas públicas com efetividade local.

Diante disso, optou-se por conduzir um estudo de base populacional em Joinville, cidade mais populosa do estado de Santa Catarina ${ }^{5}$. A ocorrência de 4.308 óbitos por causas externas no município entre 2000 e 2015 mostra a magnitude do problema, responsável por cerca de $11 \%$ do total de óbitos registrados 6 . Vale ressaltar que o Sistema de Informação de Mortalidade (SIM) municipal possui cobertura universal, boa qualidade de registros e é frequentemente auditado, sendo considerado fonte confiável de dados para estudos.

Considerando a magnitude e o aumento das mortes devido às causas externas observados nos últimos anos nesse estado e município, o presente estudo tem o objetivo de descrever o perfil das mortes por causas externas em Joinville e verificar sua tendência nos últimos anos.

\section{MÉTODO}

Trata-se de estudo observacional longitudinal retrospectivo, com análise dos óbitos por causas externas ocorridos em residentes de Joinville (SC), no período de 1 de janeiro de 2003 a 31 de dezembro de 2016.

Os dados de mortalidade relativos ao período estudado foram extraídos do Sistema de Informações sobre Mortalidade, cuja fonte é a Declaração de Óbito (DO). O registro da causa básica do óbito baseia-se no CID-10 e a identificação do grupo de causas externas, utilizou o capítulo XX - Causas externas de morbidade e de mortalidade. No presente estudo, considerou-se quatro agrupamentos de causas: acidentes de transporte terrestre, lesões autoprovocadas, agressões e outras causas.

Foram incluídos no estudo apenas os óbitos de pessoas residentes em Joinville (SC), para o qual utilizamos os filtros "Óbitos por residência" e "Município de Joinville" no SIM.

Os dados sobre os óbitos e as estimativas populacionais de 2003 a 2015 usados na elaboração dos coeficientes de mortalidade estão disponíveis no sítio eletrônico do Departamento de Informática do Sistema Único de Saúde (Datasus), através do TabNet, segundo faixa etária e sexo. Para o ano de 2016, utilizaram-se os dados da base local do SIM e a estimativa populacional do município da Secretaria Municipal de Saúde ${ }^{6}$, uma vez que esses dados não estavam disponíveis pelo Datasus no momento da coleta dos dados.

As variáveis analisadas foram sexo, idade na ocasião do óbito (em anos completos e agrupados em faixas etárias), raça/cor (preta/parda e branca), local da ocorrência do óbito (hospital, domicílio, via pública, outro), realização de necropsia, tipos de óbitos (acidentes de transporte terrestre, agressões, lesões autoprovocadas e outras causas) e fonte de informação (boletim de ocorrência, hospital, outra). Foram elaboradas planilhas pelo programa Microsoft Excel, com distribuições de frequências dos óbitos por grupo etário, sexo e ano. Calcularam-se os coeficientes de mortalidade para cada tipo de causa externa e, para dar maior estabilidade 
a esse indicador, optou-se por trabalhar com biênios. Procedeu-se à padronização dos coeficientes de mortalidade por grupo etário, pelo método direto, utilizando a população brasileira como padrão e encontraram-se resultados semelhantes, o que levou os autores e utilizarem os valores não padronizados.

$\mathrm{Na}$ análise transversal dos resultados, utilizou-se o teste do qui-quadrado para comparar as diferenças encontradas em cada faixa etária segundo o sexo e os tipos de óbitos.

$\mathrm{Na}$ análise longitudinal, para verificação da tendência dos óbitos masculinos no período analisado, adotou-se o modelo de regressão quasi Poisson ou Poisson com variância robusta. Esse modelo foi escolhido em virtude da dispersão dos dados ao longo do período analisado. As equações de tendência linear e as estatísticas de ajuste de modelo (valor de R2 ajustado e o valor de $p$ do teste $F$ de adequação do modelo) foram obtidas com o software estatístico $R$ versão $3 \cdot 3^{7}$. O nível de significância adotado foi $p<0,05$.

O estudo seguiu as recomendações éticas da Resolução n ${ }^{466 / 2012}$ do Conselho Nacional de Saúde, sendo aprovado pelo Comitê de Ética em Pesquisa da Universidade da Região de Joinville, com parecer $\mathrm{n}^{\circ} 1.871 .971$.

\section{RESULTADOS}

No período analisado, Joinville registrou 3.382 óbitos por causas externas e totalizou um coeficiente de mortalidade de 46,3 por 100 mil habitantes, evoluindo do biênio inicial ao final da série histórica, de 41,8 até 53,7 por 100 mil habitantes. A mortalidade proporcional por causas externas representou $10 \%$ do total de óbitos do período. O sexo masculino teve $83,6 \%$ dos óbitos e a faixa etária mais acometida foi de $25-39$ anos (30,5\%), seguida de $40-59$ anos $(25,5 \%)$ e de $15-24$ anos (24,8\%). Dentre os tipos de causas, os acidentes por transporte terrestre foram a principal, compreendendo $40 \%$ do total, seguidos das agressões $(30,5 \%)$ e outras causas $(17,1 \%)$. Os óbitos foram predominantemente hospitalares $(44,4 \%), 93,5 \%$ realizaram necropsias e a principal fonte de informação foram os boletins de ocorrência $(55,2 \%)$ (Tabela 1).

A faixa etária de 0-14 anos representou uma parcela pequena do total de óbitos por causas externas $(n=114 ; 3,4 \%)$. Para ambos os sexos, a principal causa de morte foram os acidentes de transportes terrestres, que corresponderam a $47,3 \%$ dos óbitos entre os meninos e $42,5 \%$ entre as meninas $(p=0,900)$. Chama atenção nesse grupo etário a ocorrência de óbitos devido a agressões ( $n=14)$ ou lesões autoprovocadas (4), mostrando a precocidade da violência que atinge especialmente os meninos (12 casos entre os 18 óbitos). O predomínio de óbitos por todos os tipos de causas entre os meninos desse grupo evidencia o início de uma tendência que vai se definir mais nitidamente nos grupos etários seguintes.

Nos grupos de 15-24 e 25-39 anos, identifica-se predomínio de dois principais tipos de óbitos por causas externas, agressões $(41,2 \%)$ seguidas de acidentes de transportes terrestres $(38,0 \%)$. Nesses grupos de jovens e adultos jovens, fica evidente o grande predomínio de ocorrências no sexo masculino $(89,1 \%)$ e, ao se comparar cada tipo separadamente entre os sexos, encontrou-se que $93,8 \%$ dos óbitos por agressões e $85,2 \%$ por acidentes de transportes terrestres ocorreram em homens, diferença significativa em comparação com as mulheres ( $p<0,001$ e $p=0,008$, respectivamente). Além disso, nesses grupos, começam a ganhar relevância os óbitos por lesões autoprovocadas (10,2\% do total de óbitos nessa faixa de idade) com predomínio no sexo masculino ( $86,3 \%)$, embora sem diferença significativa $(p=0,300)$. No sexo feminino, a maior frequência de óbitos nesses grupos etários foi por acidentes de transporte terrestre $(51,5 \%)$.

Entre os adultos de 40 a 59 anos, predominam os óbitos por acidentes de transporte terrestre (40,5\%). Para todos os tipos, houve predomínio do sexo masculino, com $81,4 \%$ dos acidentes de transporte terrestre ( $p=0,476), 88,3 \%$ das agressões $(p=0,094)$ e $76,3 \%$ das lesões autoprovocadas $(p=0,048)$. Nesse grupo etário ainda se verifica a maior proporção de óbitos por lesões autoprovocadas, representando $17,6 \%$ do total de óbitos.

Nos idosos, a tendência de predomínio de óbitos por acidentes de transporte terrestre permanece, representando 45,3\%. Da mesma forma, todos os tipos são mais frequentes nos idosos do sexo masculino, com $75,3 \%$ dos óbitos por acidentes, $79,5 \%$ por agressões e $79,2 \%$ por lesões autoprovocadas, sem diferença entre os sexos ( $(p=0,145,0,276$ e 0,136 , respectivamente). 


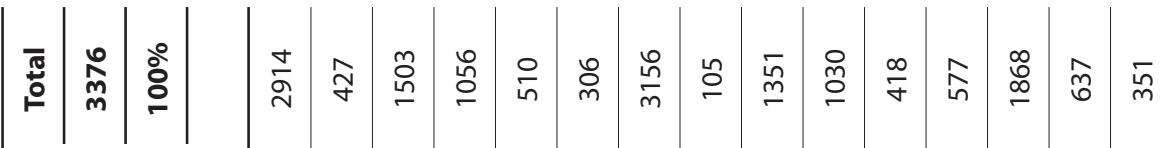

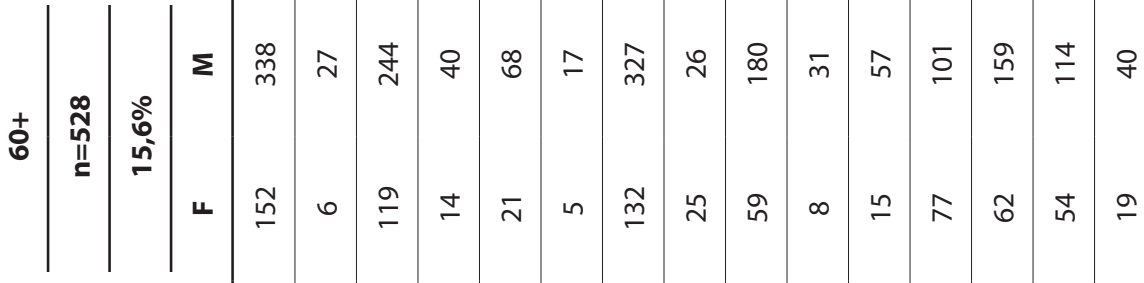

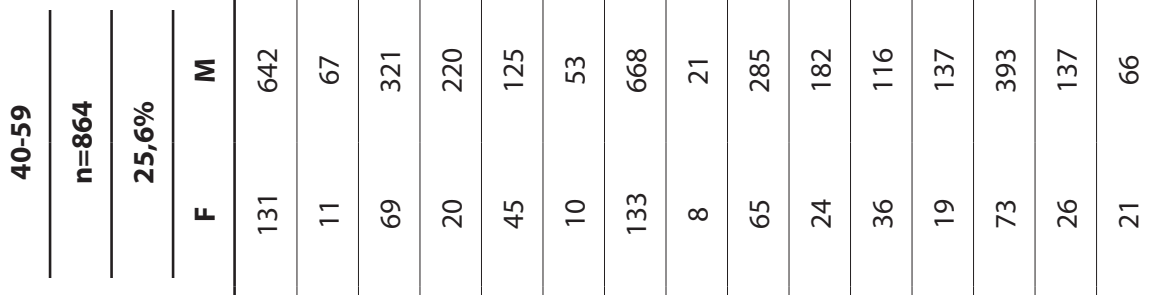

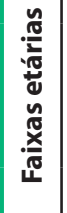

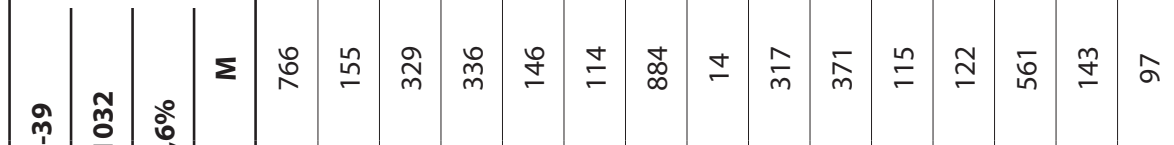

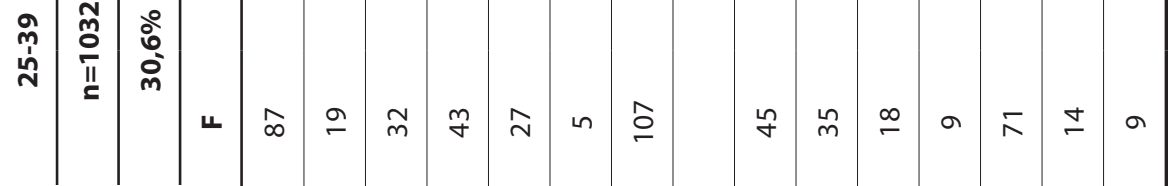
J

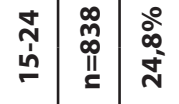

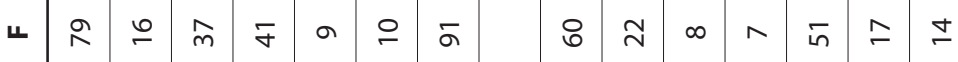

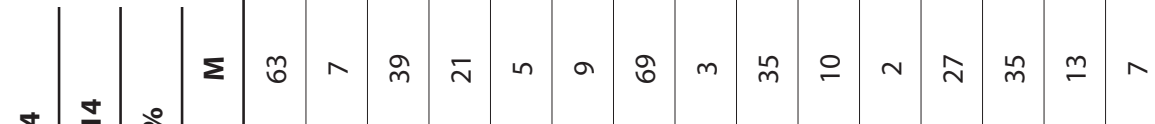

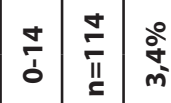

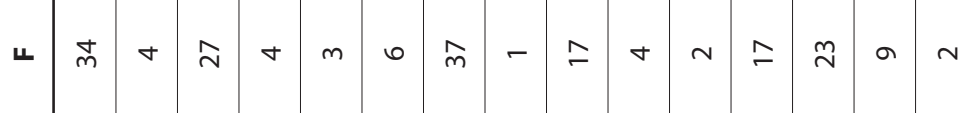

I

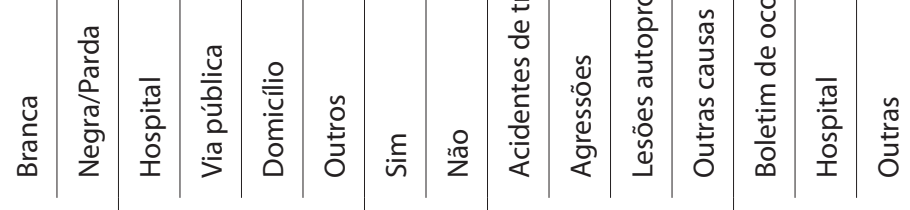

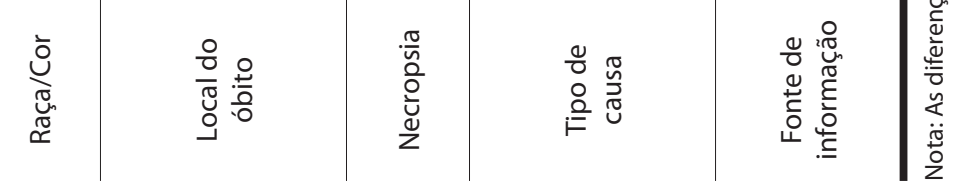


Apesar da distribuição dos óbitos por causas externas entre os sexos mostrar predomínio dos homens em todas as faixas etárias, os maiores diferenciais entre os sexos são observados em adolescentes e jovens ( 15 a 24 anos) e adultos jovens ( 25 a 39 anos), com proporções entre os homens, respectivamente, de $88,4 \%$ e $89,6 \%$ e diferença significativa em relação às mulheres $(p=0,001$ e $p<0,001$, respectivamente) (Tabela 1$)$.

A evolução temporal dos coeficientes de mortalidade por causas externas segundo tipo de óbito e sexo mostra predomínio dos acidentes de transporte terrestre em ambos os sexos, sendo superado nos últimos anos pelas agressões no sexo masculino e pelas outras causas no feminino (Figura 1).
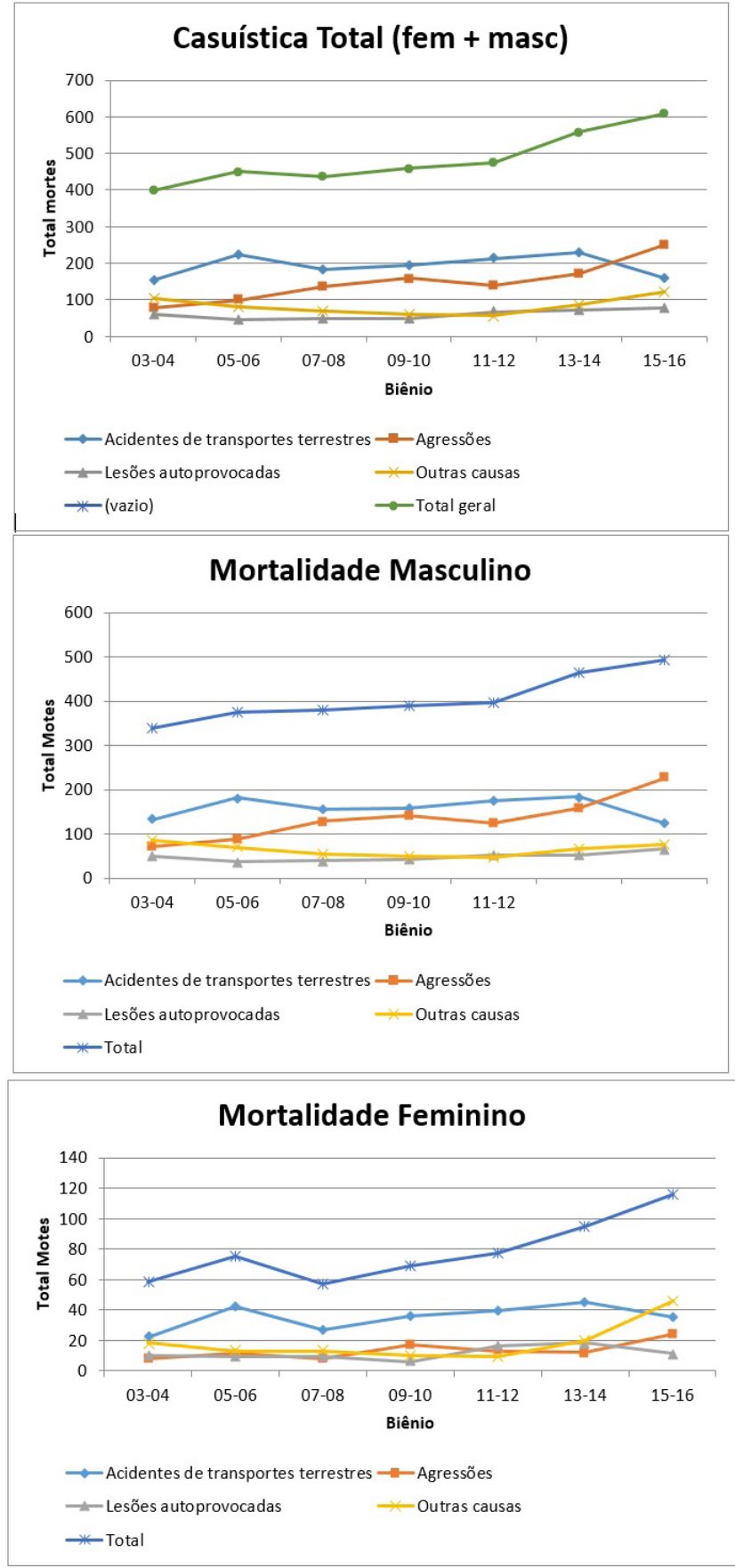

Figura 1. Coeficiente de mortalidade por causas externas segundo sexo e tipo de causa, no município de Joinville (SC), 2003 a 2016 
Os coeficientes de mortalidade conforme os tipos de causas externas nos grupos etários e sexos mostram oscilações ao longo do período, evidenciando distintos perfis para cada subgrupo (Figura 2).

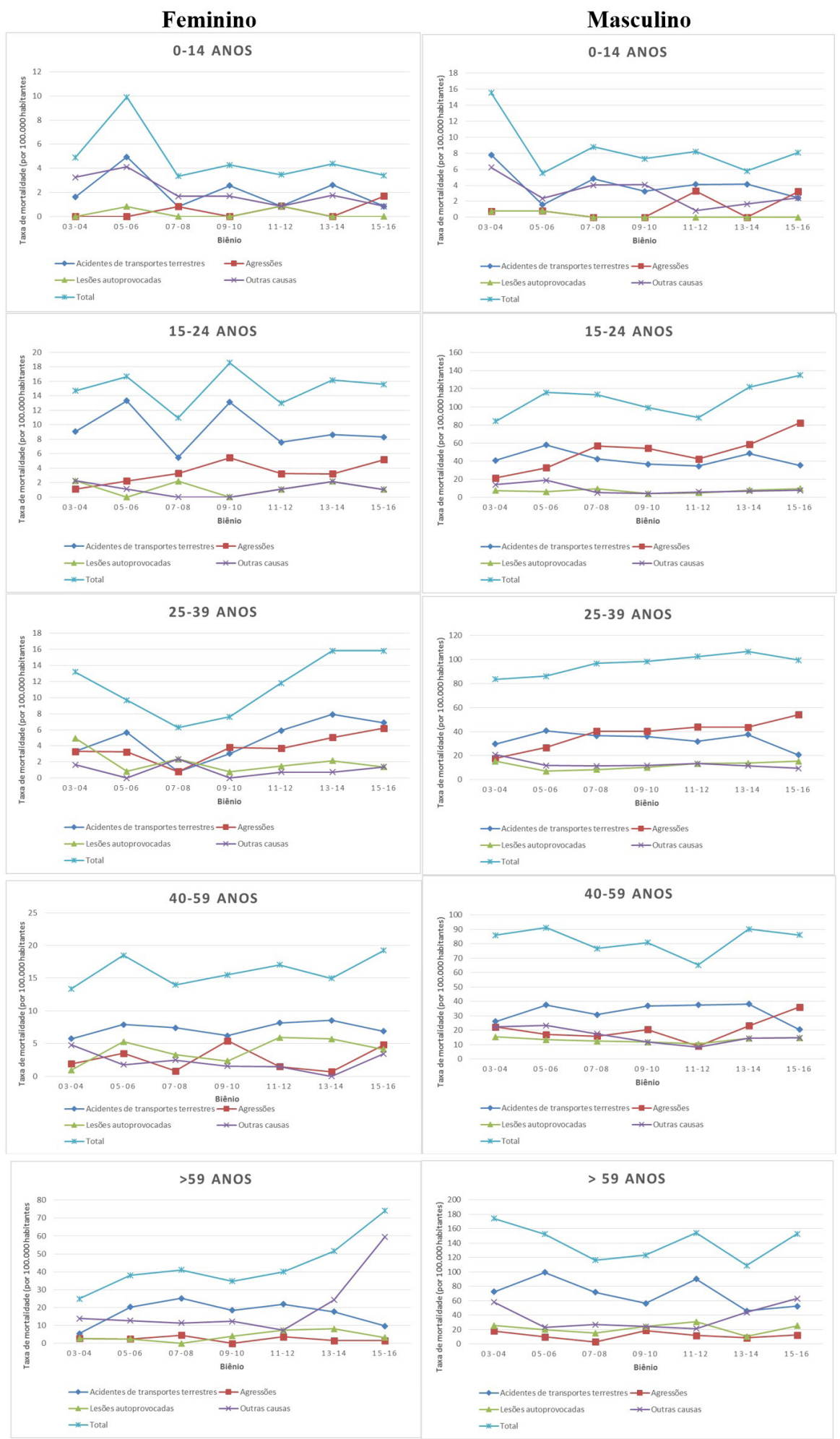

Figura 2. Coeficiente de mortalidade por grupos de causas externas segundo sexo e idade, no município de Joinville (SC), 2003 a 2016 
Ao longo do período, os óbitos por acidentes apresentaram redução significativa no grupo etário de 0 a 14 anos, tanto no sexo masculino $(p<0,001)$ quanto no feminino $(p=0,002)$ e aumento nos idosos de ambos os sexos $(p<0,001)$. Para as demais faixas etárias não se observaram mudanças significativas (Tabela 2).

Tabela 2. Análise de regressão quasipoisson - Poisson com variância robusta para os tipos de óbitos por causas externas. Joinville (SC), 2003 a 2016.

\begin{tabular}{|c|c|c|c|c|c|}
\hline & $\begin{array}{c}\text { Grupo etário } \\
\text { (em anos) }\end{array}$ & Sexo & Coeficiente & IC $_{\mathbf{9 5 \%}}$ & Valor P \\
\hline \multirow{10}{*}{ Acidentes } & $0-14$ & M & $-2,26$ & $-3,03-1,63$ & $<0,001$ \\
\hline & & $\mathrm{F}$ & $-1,38$ & $-2,26-0,67$ & 0,002 \\
\hline & $15-24$ & M & 0,43 & $-0,070,89$ & 0,089 \\
\hline & & $\mathrm{F}$ & 0,41 & $-0,200,96$ & 0,177 \\
\hline & $25-39$ & $M$ & 0,12 & $-0,370,58$ & 0,610 \\
\hline & & $\mathrm{F}$ & $-0,42$ & $-1,070,15$ & 0,180 \\
\hline & $40-59$ & $M$ & 0,10 & $-0,410,56$ & 0,703 \\
\hline & & $\mathrm{F}$ & 0,10 & $-0,490,65$ & 0,728 \\
\hline & Mais de 60 & $M$ & 0,90 & $0,391,37$ & $<0,001$ \\
\hline & & $\mathrm{F}$ & 1,09 & $0,661,50$ & $<0,001$ \\
\hline \multirow{10}{*}{$\begin{array}{l}\text { Lesões } \\
\text { autoprovocadas }\end{array}$} & $0-14$ & M & $-3,96$ & $-6,65-2,52$ & $<0,001$ \\
\hline & & $\mathrm{F}$ & $-2,43$ & $-4,69-1,10$ & 0,007 \\
\hline & $15-24$ & $M$ & $-0,31$ & $-1,040,31$ & 0,374 \\
\hline & & $\mathrm{F}$ & $-0,65$ & $-1,830,26$ & 0,225 \\
\hline & $25-39$ & M & 0,37 & $-0,170,88$ & 0,176 \\
\hline & & $\mathrm{F}$ & $-0,14$ & $-0,970,58$ & 0,712 \\
\hline & $40-59$ & $M$ & 0,50 & $-0,041,00$ & 0,068 \\
\hline & & $\mathrm{F}$ & 0,95 & $0,321,56$ & 0,005 \\
\hline & Mais de 60 & M & 0,94 & $0,331,48$ & 0,003 \\
\hline & & $\mathrm{F}$ & 0,80 & $-0,061,52$ & 0,050 \\
\hline \multirow{10}{*}{ Agressões } & $0-14$ & M & $-3,37$ & $-5,94-1,95$ & $<0,001$ \\
\hline & & $\mathrm{F}$ & $-1,88$ & $-3,04-1,01$ & $<0,001$ \\
\hline & $15-24$ & $M$ & 0,92 & $0,411,41$ & 0,001 \\
\hline & & $\mathrm{F}$ & 0,36 & $-0,260,93$ & 0,234 \\
\hline & $25-39$ & M & 0,61 & $0,031,17$ & 0,042 \\
\hline & & $\mathrm{F}$ & 0,57 & $0,061,06$ & 0,033 \\
\hline & $40-59$ & $M$ & $-0,27$ & $-1,000,37$ & 0,440 \\
\hline & & $\mathrm{F}$ & 0,07 & $-0,540,62$ & 0,814 \\
\hline & Mais de 60 & M & $-0,83$ & $-2,780,38$ & 0,282 \\
\hline & & $\mathrm{F}$ & $-0,12$ & $-1,140,68$ & 0,800 \\
\hline \multirow{10}{*}{ Outras causas } & $0-14$ & M & $-1,58$ & $-2,70-0,74$ & 0,003 \\
\hline & & $\mathrm{F}$ & $-0,66$ & $-1,211,87$ & 0,563 \\
\hline & $15-24$ & M & $-0,38$ & $-1,280,36$ & 0,368 \\
\hline & & $\mathrm{F}$ & $-1,32$ & $-7,430,99$ & 0,436 \\
\hline & $25-39$ & $M$ & 0,05 & $-0,630,67$ & 0,875 \\
\hline & & $\mathrm{F}$ & $-1,52$ & $-6,150,56$ & 0,294 \\
\hline & $40-59$ & $M$ & 0,33 & $-0,330,95$ & 0,312 \\
\hline & & $\mathrm{F}$ & $-0,63$ & $-3,581,15$ & 0,560 \\
\hline & Mais de 60 & M & 1,30 & $0,821,75$ & $<0,001$ \\
\hline & & $\mathrm{F}$ & 2,64 & $2,093,20$ & $<0,001$ \\
\hline
\end{tabular}


Tabela 2. Continuação...

\begin{tabular}{|c|c|c|c|c|c|}
\hline & $\begin{array}{c}\text { Grupo etário } \\
\text { (em anos) }\end{array}$ & Sexo & Coeficiente & IC $_{95 \%}$ & Valor P \\
\hline \multirow{10}{*}{ Total } & $0-14$ & M & $-2,45$ & $-3,27-1,80$ & $<0,001$ \\
\hline & & $\mathrm{F}$ & $-1,32$ & $-2,24-0,58$ & 0,002 \\
\hline & $15-24$ & M & 0,43 & $0,090,76$ & 0,012 \\
\hline & & $\mathrm{F}$ & 0,003 & $-0,590,67$ & 0,993 \\
\hline & $25-39$ & M & 0,31 & $-0,020,63$ & 0,063 \\
\hline & & $\mathrm{F}$ & $-0,34$ & $-0,950,21$ & 0,253 \\
\hline & $40-59$ & M & 0,078 & $-0,010,16$ & 0,665 \\
\hline & & $\mathrm{F}$ & 0,096 & $-0,470,63$ & 0,729 \\
\hline & Mais de 60 & M & 0,645 & $0,181,06$ & $<0,001$ \\
\hline & & $\mathrm{F}$ & 1,35 & $0,981,71$ & $<0,001$ \\
\hline
\end{tabular}

Com relação aos óbitos por lesões autoprovocadas, observou-se redução na faixa etária de 0 a 14 anos para ambos os sexos ( $p<0,001$ e $p=0,007$, respectivamente). Identificou-se aumento entre as mulheres de 40 a 59 anos $(p=0,005)$ e entre os homens acima de 60 anos (idosos) ( $p=0,003)$.

Os óbitos por agressões também apresentaram redução na faixa etária mais jovem (0-14 anos) para ambos os sexos $(p<0,001)$, porém observou-se aumento em adolescentes e jovens ( $15-24$ anos) masculinos ( $p=0,001)$ e adultos jovens ( $25-39$ anos) de ambos os sexos $(p=0,042$ e 0,033 , respectivamente). Os óbitos devido às outras causas tiveram redução na faixa etária de 0 a 14 anos e sexo masculino $(p=0,003)$ e aumento em idosos de ambos os $\operatorname{sexos}(p<0,001)$.

Considerando todos os óbitos por causas externas, no período estudado observou-se redução significativa apenas em idades precoces ( $0-14$ anos) para ambos os sexos $(p<0,001$ e $p=0,002$, respectivamente para homens e mulheres), aumento entre homens nos grupos etários de adolescentes e jovens ( $15-24$ anos) $(p=0,012)$ e idosos de ambos os sexos $(p<0,001)$.

\section{DISCUSSÃO}

O coeficiente de mortalidade por causas externas em Joinville situa-se abaixo do estado de Santa Catarina e das demais regiões brasileiras ${ }^{4}$. Entretanto, é preocupante o aumento observado de $29 \%$ nos coeficientes (considerando-se os biênios extremos), mesmo que a mortalidade proporcional por essas causas tenha se mantido constante, ao redor de $10 \%$, durante o período estudado. Esse resultado é semelhante a outras localidades nacionais onde se observa aumento expressivo nos coeficientes nos últimos anos ${ }^{8-16}$.

A principal limitação do estudo refere-se à qualidade dos dados do SIM, havendo possibilidade de haver sub-registro das informações sobre a causa básica dos óbitos ou elevado percentual de óbitos por intenção determinada, com consequente subestimativa dos coeficientes de mortalidade. Checagens rotineiras realizadas pela Vigilância Epidemiológica no município estudado foram instituídas desde 2012 com o objetivo de melhorar a qualidade dos registros e reduzir tais problemas. Em nosso estudo, acreditamos que a exclusão de apenas seis casos sem registros de idade não tenha influenciado a análise dos resultados aqui apresentados em virtude do tamanho da casuística. De todo modo, é importante conhecer as limitações dos registros utilizados e contribuir para sua melhoria continuamente.

Em nosso estudo, foram identificadas diferenças no perfil da mortalidade conforme o sexo e o grupo etário analisado. Entre os homens, o maior coeficiente de mortalidade foi no grupo de 25 a 39 anos (96,7 por 100 mil) com predomínio das agressões e nas mulheres foi no grupo entre 40 e 59 anos (16,2 por 100 mil) devido a acidentes por transporte. Chama atenção o predomínio do sexo masculino em todos os grupos etários, com uma média de razão de sexo de 5:1, principalmente no grupo etário de 25-39 anos, no qual alcança 8,6:1. 
Esse resultado já foi relatado em outros estudos brasileiros e mundiais ${ }^{11,12,14,15,17,18}$ e evidencia um comportamento do gênero masculino determinado por fatores culturais e sociais que definem os modos particulares de vivenciar a masculinidade, especialmente pela associação do masculino à necessidade de se expor ao risco.

Em relação aos tipos de causa externa, o predomínio dos acidentes por transporte terrestre seguido das agressões é uma tendência observada nacionalmente ${ }^{9,12,13}$. Ambos os tipos de mortes ocorrem precocemente, trazendo importantes consequências sociais e econômicas com expressivo impacto econômico para o setor saúde e a sociedade como um todo. Ao serem de eventos passíveis de prevenção, as ações de intervenção ganham especial relevância.

Sabe-se que o aumento dos acidentes de transporte terrestre acompanha o processo de desenvolvimento e a maior circulação de pessoas e mercadorias. Essa relação é observada em estudos que mostram aumento da mortalidade por acidentes de transporte terrestre em localidades que apresentam índices compatíveis com melhor grau de desenvolvimento, tais como maiores taxas de crescimento demográfico, maiores níveis de renda e menores percentuais de analfabetos ${ }^{17}$. Dentre as políticas públicas para enfrentar o aumento da mortalidade por acidentes de transporte terrestre, a implantação do Código de Trânsito Brasileiro (CTB) em 1998 produziu impacto importante nas tendências dessa mortalidade nas regiões brasileiras, conforme mostra o Ministério da Saúde, com destaque para a redução nas regiões Sul, Sudeste e Centro-Oeste, na maior parte das faixas etárias e em ambos os sexos estudados $^{19}$. No Brasil, entre 1980 e 2005, observou-se inversão na tendência de incremento da mortalidade por acidentes de trânsito após a implantação do CTB, constatando-se uma redução imediata de mais de 5 mil mortes. Esse impacto foi desigual nas diversas regiões estudadas ${ }^{13}$. Resultados similares foram encontrados em diversos municípios brasileiros especialmente após campanhas lançadas pelas áreas de Saúde e Seguridade Social, bem como pela promulgação da Lei Seca em $2008^{11,16}$. Em Joinville, a mortalidade por acidentes de transporte terrestre teve discreta tendência de queda no último biênio, embora tenha sido significativa apenas entre as crianças e adolescentes. Ressalte-se que a mortalidade por acidentes apresentou tendência significativa de elevação em idosos de ambos os sexos. É possível que o elevado grau de desenvolvimento desse município, com grande dinamismo do setor produtivo industrial, e a necessidade de escoamento das mercadorias produzidas, especialmente por veículos de transporte terrestre, seja um fator que dificulte a redução da mortalidade esperada em virtude das políticas adotadas nos últimos anos. Entretanto, há necessidade de mais estudos na localidade estudada, qualitativos e quantitativos, com dados mais detalhados sobre o comportamento de risco das pessoas em diferentes fases da vida, os subtipos de morte de cada grupo etário com mais detalhes para clarificar melhor as causas associadas direta e indiretamente ao evento, e outros. Já, o aumento da mortalidade por acidentes em idosos de ambos os sexos em Joinville chama atenção para a questão da relação entre o processo de envelhecimento e a aptidão para dirigir. O aumento da longevidade da população do município, atualmente em 78,3 anos $^{6}$, acima da média nacional, se por um lado é um indicador positivo associado ao maior grau de desenvolvimento, por outro, aponta uma preocupação relativa à perda da capacidade de dirigir e a consequente necessidade dos órgãos de trânsito aprimorarem a análise da capacidade do idoso para dirigir, com avaliação neuropsicológica para a identificação de deficiências auditivas e visuais e de déficits cognitivos, através de instrumentos específicos para o rastreamento de demência.

Em nosso estudo, o grupo de 0-14 anos apresentou tendência significativa de redução na mortalidade por acidentes de transporte terrestre, com queda de $46 \%$ considerando-se os biênios extremos. No Brasil, dados do Datasus mostram evolução semelhante entre 2001 e 2015, com redução de $31,5 \%^{3}$. Provavelmente, as melhorias da segurança nos meios de transporte e no trânsito, a implantação de mecanismos adequados de atendimento às vítimas e a estruturação da retaguarda hospitalar para atenção a pacientes com lesões muito graves verificadas nos últimos anos tiveram impacto sobre esse indicador.

A análise da mortalidade por agressões remete à questão da violência e seu aumento evidencia um dos maiores problemas de saúde pública na realidade contemporânea de nosso país, que adquire características próprias, conforme as circunstâncias sociais, culturais 
e políticas específicas de cada região ${ }^{16}$. Sabe-se que a violência está diretamente relacionada com o aumento das disparidades socioeconômicas, entretanto a elevação da mortalidade por agressões entre os jovens e adultos jovens do sexo masculino observada em Joinville aponta que o elevado desenvolvimento humano e social $(\mathrm{IDH}=0,809)$ e a menor iniquidade dessa localidade ${ }^{20}$ não foram suficientes para atenuar essa tendência nacional. Para se elaborar estratégias para redução da violência é necessário compreender os fatores envolvidos em sua complexa determinação social, especialmente o crime organizado ligado ao tráfico de drogas, hoje disseminado por todo território nacional, e as questões relacionadas ao gênero masculino.

$\mathrm{O}$ aumento das lesões autoprovocadas em mulheres adultas e homens idosos aqui observado chama atenção para um problema atual de saúde pública que é o crescimento dos suicídios em pessoas de idade mais avançada, especialmente em idosos. $\mathrm{O}$ aumento da longevidade torna necessário um melhor entendimento a respeito dos processos biológicos, psicológicos e sociais que exacerbam o desejo de autodestruição à medida que a idade aumenta. Além de estudos específicos sobre traços de personalidade, fatores associados a doenças físicas, estresse e suas implicações médicas, psiquiátricas e neuropsicológicas, é preciso aprimorar os cuidados de saúde desde o diagnóstico da depressão, que é o fator desencadeador mais relevante, até as ações que ajudem a reduzir sofrimentos e dependências e ações sociais que auxiliem os idosos a interagirem com sua comunidade e manterem a dignidade pessoal no final da vida ${ }^{21}$.

Nesse mesmo grupo etário, nosso estudo identificou aumento significativo de mortes devido a outras causas externas, incluindo os traumatismos acidentais. Tendência semelhante foi observada em outros estudos, revelando a exposição dos idosos aos acidentes ${ }^{10,14} \mathrm{e}$ indicando a necessidade de os profissionais de saúde conhecerem as características da população idosa e os riscos aos quais ela se expõe, para assim atuarem preventivamente na redução de atropelamentos, quedas e demais acidentes. Nesse sentido, as ações de prevenção secundária ganham importância, objetivando a melhora na acuidade visual, na baixa densidade mineral óssea, na baixa atividade física e na fraqueza muscular, entre outras.

A elevada longevidade da população de Joinville e o aumento da mortalidade por causas externas chamam atenção para a necessidade de seu enfrentamento por meio da implantação de políticas específicas voltadas para esse grupo etário, considerando as especificidades na determinação dos diversos tipos de mortes compreendidos nesse grupo.

Na localidade estudada, ações no âmbito da saúde pública devem incluir a vigilância de acidentes de transporte e agressões, principais causas externas de morte observadas, e fornecer informações para subsidiar os gestores na formulação de estratégias locais. Merece destaque a melhoria na articulação entre o atendimento pré-hospitalar e a assistência hospitalar de urgência e emergência. No caso das mortes por acidentes de transporte terrestre, a promoção de ações intersetoriais, envolvendo a saúde, a educação e os órgãos ligados ao controle do trânsito para dar continuidade à implantação do Código de Trânsito Brasileiro com foco no processo educativo dos condutores e pedestres; uso de cintos de segurança, transporte adequado de crianças em veículos, respeito aos limites de velocidade e o enfrentamento de novas realidades, como uso do álcool e aumento das motocicletas, são algumas das medidas a serem tomadas. No caso das agressões, é necessário que a violência seja considerada como um problema de toda a sociedade e abordada em interação com os diferentes setores, como as áreas da Justiça, da segurança, da educação e com os movimentos sociais. Iniciativas voltadas para a prevenção, atenção e reabilitação das vítimas diretas e indiretas das agressões letais e não letais são necessárias e urgentes diante do recrudescimento da violência em nossa sociedade. Não se pode deixar de mencionar que o protagonismo do setor da saúde em Joinville pode produzir impacto na melhora da atenção às vítimas, desenvolvendo ações no âmbito da formação e da educação continuada dos profissionais de saúde, com a inclusão do tema como uma das prioridades, desde os Programas Saúde da Família até os demais níveis hierárquicos da rede de atenção às urgências e emergências.

No período analisado, Joinville registrou 3.382 óbitos por causas externas e totalizou um coeficiente de mortalidade de 46,3 por 100 mil habitantes, sendo $83,6 \%$ homens. O coeficiente foi de 41,8 por 100 mil habitantes no biênio inicial da série histórica e aumentou 
progressivamente até atingir 53,7 por 100 mil habitantes no final, totalizando uma elevação de $53 \%$. Dentre os tipos de causas, os acidentes por transporte terrestre foram o principal, compreendendo $40 \%$ do total, seguidos das agressões $(30,5 \%)$ e outras causas $(17,1 \%)$. Os óbitos foram predominantemente hospitalares (44,4\%), 93,5\% realizaram-se necropsias e a principal fonte de informação foram os boletins de ocorrência $(55,2 \%)$. Ao longo do período observaram-se variações segundo sexo, grupo etário e tipo, com redução no grupo etário de 0-14 anos e aumento nos grupos de 15-24, 25-39 anos e acima de 60 anos. A elevação da mortalidade por agressões entre os jovens e adultos jovens do sexo masculino observada em Joinville aponta que o elevado desenvolvimento humano e social (IDH=0,809) e a menor iniquidade dessa localidade não foram suficientes para atenuar essa tendência nacional. $\mathrm{O}$ aumento da mortalidade em idosos torna evidente a maior vulnerabilidade desse grupo, cujas mudanças de comportamento têm ocasionado maior exposição a riscos Tais grupos devem tornar-se alvo de políticas públicas.

\section{REFERÊNCIAS}

1. World Health Organization. World Report on Violence and Health [Internet]. Genebra: World Health Organization; 2002 [citado em 2017 Out 10]. Disponível em: http://apps.who.int/iris/ bitstream/10665/42495/1/9241545615_eng.pdf

2. World Health Organization. Injuries and violence: the facts [Internet]. Genebra: World Health Organization; 2010 [citado em 2017 Out 10]. Disponível em: http://www.who.int/violence_injury_prevention/key_facts/en/

3. Brasil. Ministério da Saúde. Mortalidade-Brasil. TabNet [Internet]. 2017 [citado em 2017 Jul 4]. Disponível em: http://tabnet.datasus.gov.br/cgi/tabcgi.exe?sim/cnv/obt10uf.def

4. Rede interagencial de informações para saúde. Indicadores e Dados Básicos para a Saúde no Brasil (IDB) [Internet]. 2017 [citado em 2017 Jul 4]. Disponível em: http://www.ripsa.org.br/vhl/indicadores-e-dadosbasicos-para-a-saude-no-brasil-idb/

5. Instituto Brasileiro de Geografia e Estatística. Histórico do município [Internet]. Rio de Janeiro; 2010 [citado em 2017 Jul 4]. Disponível em: http://www.cidades.ibge.gov.br/painel/historico.php?lang=\&codmun=4 20910\&search=santa-catarina\%7Cjoinville\%7Cinfograficos:-historico

6. Instituto de Pesquisa e Planejamento para o Desenvolvimento Sustentável de Joinville. Cidade em dados 2016. Joinville: IPPUJ [Internet]. 2017 [citado em 2017 Nov 10]. Disponível em: https://www.joinville.sc.gov. br/wp-content/uploads/2016/01/Joinville-Cidade-em-Dados-2016.pdf

7. R Core Team. R: A language and environment for statistical computing [Internet]. Viena, Áustria: R Foundation for Statistical Computing; 2017 [citado em 2017 Jul 12]. Disponível em: http://www.r-project. org/

8. Costa JSD, Giraldi MC, Carret MLV, Ferreira AMTB, Strauch ES, Moraes M, et al. Evolução da mortalidade por causas externas no município de Pelotas e no estado do Rio Grande do Sul, Brasil, 1996-2009. Epidemiol Serv Saude. 2013;22(2):215-24. http://dx.doi.org/10.5123/S1679-49742013000200003.

9. Martins CB, Andrade SM. Epidemiologia dos acidentes e violências entre menores de 15 anos em município da região sul do Brasil. Rev Lat Am Enfermagem. 2005;13(4):530-7. http://dx.doi.org/10.1590/ S0104-11692005000400011. PMid:16211177.

10. Mathias TA, Jorge MH, Andrade O. Morbimortalidade por causas externas na população idosa residente em município da região sul do Brasil. Rev Lat Am Enfermagem. 2006;14(1):17-24. http://dx.doi.org/10.1590/ S0104-11692006000100003. PMid:16532235.

11. Gomes LP, Melo ECP. Distribuição da mortalidade por acidentes de trânsito no município do Rio de Janeiro. Esc Anna Nery. 2007;11(2):289-95. http://dx.doi.org/10.1590/S1414-81452007000200016.

12. Souza MFM, Malta DC, Conceição GMS, Silva MMA, Gazal-Carvalho C, Morais OL No. Análise descritiva e de tendência de acidentes de transporte terrestre para políticas sociais no Brasil. Epidemiol Serv Saude. 2007;16(1):33-44.

13. Duarte EC, Tauil PL, Duarte E, Sousa MC, Monteiro RA. Mortalidade por acidentes de transportes terrestre e homicídios em homens jovens das capitais das Regiões Norte e Centro-Oeste do Brasil, 1980-2005. Epidemiol Serv Saude. 2008;17(1):7-20.

14. Freire GA et al. Mortalidade por causas externas em idosos no Paraná, Brasil de 2001-2010. UNOPAR Cient Ciênc Biol Saúde. 2013;15(2):161-7. 
15. Brasil. Instituto Brasileiro de Geografia e Estatística. Tábua completa de mortalidade para o Brasil - 2015 [Internet]. Rio de Janeiro: IBGE; 2016 [citado em 2017 Out 10]. Disponível em: ftp://ftp.ibge.gov.br/Tabuas_ Completas_de_Mortalidade/Tabuas_Completas_de_Mortalidade_2015/tabua_de_mortalidade_analise. pdf

16. Barreto MD, Teston EF, Latorre MD, Mathias TA, Marcon SS. Mortalidade por acidentes de trânsito e homicídios em Curitiba, Paraná, 1996-2011. Epidemiol Serv Saude. 2016;25(1):95-104. PMid:27861682.

17. Duarte EC, Schneider MC, Paes-Sousa R, Ramalho WM, Sardinha LMV, Silva JB Jr, et al. Epidemiologia das desigualdades em saúde no Brasil: um estudo exploratório. Brasília: Organização Pan-Americana da Saúde; 2002.

18. Laurenti R, Jorge MHPM, Gotlieb SLD. Perfil epidemiológico da morbi-mortalidade masculina. Cien Saude Colet. 2005;10(1):35-46. http://dx.doi.org/10.1590/S1413-81232005000100010.

19. Brasil. Ministério da Saúde. Secretaria de Vigilância em Saúde. Saúde Brasil, 2004: uma análise da situação de saúde [Internet]. Brasília, DF: Ministério da Saúde; 2004 [citado em 2017 Nov 10]. Disponível em: http:// bvsms.saude.gov.br/bvs/publicacoes/saude_brasil_2004.pdf

20. Gawryszewski VP, Costa LS. Homicídios e desigualdades sociais no Município de São Paulo. Rev Saude Publica. 2005;39(2):191-7. http://dx.doi.org/10.1590/S0034-89102005000200008. PMid:15895137.

21. Minayo MCS, Cavalcante FG. Suicídio entre pessoas idosas: revisão da literatura. Rev Saude Publica. 2010;44(4):750-7. http://dx.doi.org/10.1590/S0034-89102010000400020. PMid:20676565. 